\title{
SOME LINEAR SUBORDINATION RESULTS FOR CLASSES OF UNIVALENT FUNCTIONS
}

\author{
ROBERT B. BYERS
}

ABSTRACT. In this note we determine necessary and sufficient conditions on complex numbers $\lambda$ and $\mu$ such that $\lambda z /\left(1-\mu a_{2} z\right)$ is subordinate to $f(z)=z+a_{2} z^{2}+\cdots$ for all functions $f$ in certain classes of univalent functions.

1. Introduction. Let $S$ denote the class of functions $f(z)=z+a_{2} z^{2}+\cdots$ analytic and univalent in the unit disk $E=\{z:|z|<1\}$. If $f$ is in $S$ and $g$ is analytic in $E$ with $g(0)=0$ and $g(E)$ is a subset of $f(E)$, then $g$ is said to be subordinate to $f$, and $f$ is called a univalent majorant of $g$. We express this by writing $g(z) \prec f(z)$.

Let $K$ and $S^{*}$ denote the convex and starlike subclasses, respectively, of $S$. The well-known result that for each $f$ in $K, f(E)$ contains the disk $\{z:|z|<1 / 2\}$ is equivalent to the statement $z / 2 \prec f(z)$. Similarly, the Koebe " $1 / 4$ theorem" implies $z / 4 \prec f(z)$ for all $f$ in S. T. Basgoze, J. L. Frank, and F. R. Keogh [1] examined necessary and sufficient conditions on complex numbers $\lambda$ and $\mu$ such that $z / 2 \prec \lambda z+\mu a_{2} z^{2} \prec f(z)$ for $f(z)=z+a_{2} z^{2}+\cdots$ in $K$. Keogh [4] also examined the question of $z / 4 \prec \lambda z+\mu a{ }_{2} z^{2} \prec f(z)$ for $f$ in $s^{*}$.

In this paper we answer similar questions in the classes $K, S^{*}$, and $S$ for functions of the form $\lambda z /\left(1-\mu a_{2} z\right)$. Since the functions $z / 4, z / 2$, and $\lambda z /\left(1-\mu a{ }_{2} z\right)$ map $E$ onto open disks (or half planes), the various subordinations obtained are equivalent to covering theorems for functions in the classes involved.

2. Convex functions. Before stating the the orem for the class of convex functions, we present the following lemma necessary for its proof.

Lemma 1. For all $f(z)=z+a_{2} z^{2}+\cdots$ in $K, f(E)$ contains the disk $\left|w-3 \bar{a}_{2} / 2\left(4-\left|a_{2}\right|^{2}\right)\right|<3 /\left(4-\left|a_{2}\right|^{2}\right)$.

Received by the editors July 14, 1973 and, in revised form, November 19, 1973. AMS (MOS) subject classifications (1970). Primary 30A26; Secondary 30A32.

Key words and phrases. Subordination, covering theorem, univalent function, starlike, convex. 
Proof. If the function $f(z)=z+a_{2} z^{2}+\cdots$ is in $S$, and $c$ is an omitted value of $f(E)$, then the function

$$
g(z)=\frac{c f(z)}{c-f(z)}=z+\left(a_{2}+\frac{1}{c}\right) z^{2}+\cdots
$$

is in $S$. This implies [3] the inequality

$$
\left|a_{2}+1 / c\right| \leq 2
$$

If the function $f(z)=z+a_{2} z^{2}+\cdots$ is in $K$, and $c$ is an omitted value of $f(E)$, then the function

$$
g(z)=f(z)-\frac{f^{2}(z)}{2 c}=z+\left(a_{2}-\frac{1}{2 c}\right) z^{2}+\cdots
$$

is in $S$ [5], and $c / 2$ is an omitted value of $g(E)$. Applying (1) to (2), we obtain $\left|a_{2}+3 / 2 c\right| \leq 2$. The function $f(z)=z /(1-z)$ shows that the above inequality is sharp. The lemma now follows. ${ }^{1}$

Theorem 1. Let $\lambda$ and $\mu$ be complex numbers. Then

$$
z / 2 \prec \lambda z /\left(1-\mu a_{2} z\right) \prec f(z)
$$

for all $f(z)=z+a_{2} z^{2}+\cdots$ in $K$ if and only if $\lambda=1 / 2+1 / 2 \mu, 0 \leq|\mu| \leq 1 / 2$.

Proof. Without loss of generality, we can assume $\lambda \geq 0$. Let $f(z)$ be the function $z /(1-z)$. The function $|\lambda z /(1-\mu z)|$ has a minimum value of $\lambda /(1+|\mu|)$ on $|z|=1$. If the subordinations hold, we then have $\lambda /(1+|\mu|)$ $\geq 1 / 2$. Also for real $x,-1<x<0$,

$$
x /(1-x) \leq \lambda x /|1-\mu x| \leq x / 2 \text {. }
$$

Allowing $x$ to approach -1 , we obtain $\lambda /|1+\mu|=1 / 2$. This implies $\lambda=1 / 2+$ $1 / 2 \mu$ and $\mu=|\mu| \geq 0$.

If the function $f$ is in $K$, then so is the function

$$
g(z, \xi)=\frac{f[(z+\xi) /(1+\bar{\xi} z)]-f(\xi)}{f^{\prime}(\xi)\left(1-|\xi|^{2}\right)}=z+A_{2} z^{2}+\cdots, \quad|\xi|<1,
$$

where

${ }^{1}$ The author wishes to thank the referree for suggesting this simplification of the original proof. 


$$
A_{2}=\frac{f^{\prime \prime}(\xi)}{2 f^{\prime}(\xi)}\left(1-|\xi|^{2}\right)-\bar{\xi}
$$

If $f(z)=\tan ^{-1} z$ and $0<\xi<1$, then $A_{2}=-2 \xi /\left(1+\xi^{2}\right)$. If the subordinations hold for all $f$ in $K$, we then have

$$
\frac{z}{2} \prec \frac{(1 / 2+1 / 2 \mu) z}{1+2 \xi \mu z /\left(1+\xi^{2}\right)} \prec g(z, \xi),
$$

where

$$
g(z, \xi)=\frac{1+\xi^{2}}{1-\xi^{2}}\left\{\tan ^{-1} \frac{z+\xi}{1+\xi z}-\tan ^{-1} \xi\right\} .
$$

If $z$ is real, $g(z, \xi)$ is an increasing function of $z$. Letting $z=1$, we obtain the inequality

$$
\frac{1}{2} \leq \frac{1 / 2+1 / 2 \mu}{1+2 \xi \mu /\left(1+\xi^{2}\right)} \leq \frac{1+\xi^{2}}{1-\xi^{2}}\left(\frac{\pi}{4}-\tan ^{-1} \xi\right) .
$$

From this we obtain the inequality

$$
\mu\left[\left(1-\xi^{2}\right)-2 \xi\left(\frac{\pi}{2}-2 \tan ^{-1} \xi\right)\right] \leq\left(1+\xi^{2}\right)\left(\frac{\pi}{2}-2 \tan ^{-1} \xi\right)-\left(1-\xi^{2}\right)
$$

For $0<\xi<1,\left(1-\xi^{2}\right)-2 \xi\left(\pi / 2-2 \tan ^{-1} \xi\right)$ is greater than zero. Thus we have

$$
\mu \leq \frac{\left(1+\xi^{2}\right)\left(\pi / 2-2 \tan ^{-1} \xi\right)-\left(1-\xi^{2}\right)}{1-\xi^{2}-\pi \xi+4 \xi \tan ^{-1} \xi}
$$

Letting $\xi$ approach 1 , we obtain $\mu \leq 1 / 2$.

For the remainder of the proof we may assume without loss of generality that $a_{2} \geq 0$. For $a_{2} \leq 1,0 \leq \mu \leq 1$, the images of $E$ under $(1+\mu) z /\left(2-2 \mu a_{2} z\right)$ are nested. Thus we need only show that $3 z /\left(4-2 a_{2} z\right)<f(z)$. Lemma 1 completes the proof.

3. Starlike and univalent functions. The following lemma due to S. D. Bernardi [2] is used in the proof of the theorem for the univalent case.

Lemma 2. If $f(z)=z+a_{2} z^{2}+\cdots$ is in $S$, then $z /\left(2-a_{2} z\right) \prec f(z)$.

Theorem 2. Let $\lambda$ and $\mu$ be complex numbers. Then 


$$
z / 4<\lambda z /\left(1-\mu a_{2} z\right)<f(z)
$$

for all $f(z)=z+a_{2} z^{2}+\cdots$ in $S$ if and only if $\lambda=1 / 4+1 / 2 \mu, 0 \leq|\mu| \leq 1 / 2$.

Proof. Without loss of generality we can assume $\lambda \geq 0$. Suppose that for all $f(z)=z+a_{2} z^{2}+\cdots$ in $S$ we have $z / 4<\lambda z /\left(1-\mu a_{2} z\right)<f(z)$. Let $f(z)$ be the Koebe function $z /(1-z)^{2}$. As in the proof of Theorem 1, we obtain $\lambda=1 / 4+1 / 2 \mu$ and $\mu=|\mu| \geq 0$.

Next let $f(z)$ be the function $z /\left(1-z^{2}\right)=z+z^{3}+\cdots$. Letting $z=i y$, $0<y<1$, we have

$$
y / 4 \leq(1 / 4+1 / 2 \mu) y \leq y /\left(1+y^{2}\right)
$$

Letting $y$ approach 1 , we obtain $\mu \leq 1 / 2$.

For the remainder of the proof we may assume without loss of generality that $a_{2} \geq 0$. For $a_{2} \leq 2,0 \leqq \mu \leq 1 / 2$, the images of $E$ under $(1+2 \mu) z /\left(4-4 \mu a_{2} z\right)$ are nested. Lemma 2 completes the proof.

Since the functions $z /(1-z)^{2}$ and $z /\left(1-z^{2}\right)$ are starlike, Theorem 2 cannot be improved for the class $S^{*}$.

\section{REFERENCES}

1. T. Basgoze, J. L. Frank and F. R. Keogh, On convex univalent functions, Canad. J. Math. 22 (1970), 123-127. MR 41 \#1983.

2. S. D. Bernardi, Circular regions covered by schlicht functions, Duke Math. J. 32 (1965), 23-36. MR 30 \#2135.

3. W. K. Hayman, Multivalent functions, Cambridge Tracts in Math. and Math. Phys., no. 48, Cambridge Univ. Press, Cambridge, 1958, p. 3. MR 21 \#7302.

4. F. R. Keogh, A strengthened form of the $1 / 4$ theorem for starlike univalent functions, Math. Essays Dedicated to A. J. MacIntyre, Ohio Univ. Press, Athens, Ohio, 1970, pp. 201-211. MR 43 \# 496.

5. G. Sansone and J. Gerretsen, Lectures on the theory of functions of a complex variable. II: Geometric theory, Wolters-Noordhoff, Groningen, 1969, p. 198. MR $41 \# 3714$.

DEPARTMENT OF MATHEMATICS, OHIO STATE UNIVERSITY, MANSFIELD, OHIO 44906 